\title{
Hermite Solution of Bagley-Torvik Equation of Fractional Order
}

\author{
Tamour Zubair ${ }^{1,2}$, Marriam Sajjad ${ }^{3}$, Rehmatullah Madni², Amna Shabir ${ }^{2}$ \\ ${ }^{1}$ Department of Applied Mathematics and Statics, Institute of Space and Technology, Islamabad, Pakistan \\ ${ }^{2}$ Department of Mathematics, University of Sargodah, Lyallpur Campus, Faisalabad, Pakistan \\ ${ }^{3}$ Department of Mathematics, University of Lahore, Lahore, Pakistan \\ Email: *Tamourzubair@hotmail.com
}

How to cite this paper: Zubair, T., Sajjad, M., Madni, R. and Shabir, A. (2017) Hermite Solution of Bagley-Torvik Equation of Fractional Order. International Journal of Modern Nonlinear Theory and Application, 6, 104-118

https://doi.org/10.4236/ijmnta.2017.63010

Received: May 8, 2017

Accepted: August 11, 2017

Published: August 14, 2017

Copyright $\odot 2017$ by authors and Scientific Research Publishing Inc. This work is licensed under the Creative Commons Attribution International License (CC BY 4.0).

http://creativecommons.org/licenses/by/4.0/ Numerical analysis is the study of set of rules that use numerical estimation for c) (i) Open Access

\section{Keywords}

MAPLE 13, Bagley-Torvik Equations, Hermite Polynomials, Fractional Calculus

\section{Introduction}

\begin{abstract}
In this paper, a new methodology of fractional derivatives based upon Hermite polynomial is projected. The fractional derivatives are demonstrated according to Caputo sense. Hermite collocation technique is introduced to express the definite results of Bagley-Torvik Equations. The appropriateness and straightforwardness of numerical plan is presented by graphs and error tables.
\end{abstract} the problems of mathematical analysis as distinguished from discrete mathematics. Fractional differential equations are operational and most effective tool to describe different physical phenomena such as rheology, diffusion processes, damping laws, and so on. Many technics have been delegated to solve differential equation of fractional order. Different structures are used to resolve the issues of nonlinear physical models of fractional orders like Finite element method [1], Finite difference method [2], differential transformation method [3] [4], Adomian's decomposition method [5] [6] [7], variational iteration method [8] [9] [10], Homotopy perturbation technique [11], Zubair decomposition method (ZDM) [12], (G'/G)-expansion method [13], (U'/U)-expansion method [14], Uexpansion method [15], Fractional sub numerical announcement method [16] [17], Legendre wavelets technique [18], Chebyshev wavelets framework [19] [20] [21], Haar wavelets schema [22], Legendre Method [23], Chebyshev strategy [24], Jacobi polynomial scheme [25] and collocation scheme [26] [27] [28] [29]. All 
the mentioned approaches have certain limitations like excessive computational work, less efficiency to tackle nonlinearity and divergent solution due to which many issues arise. All these disputes can be fixed with the help of orthogonal polynomials, which is a vital thought in close estimation and structures. These orthogonal polynomials are the reason of powerful strategies of spectral methods [30] [31] [32]. Starting late, Khader [33] displayed a capable numerical procedure for enlightening the fractional order physical problems using the Chebyshev polynomials. In the [34] two Chebyshev spectral frameworks for measuring multi-term fractional problems are displayed. The author (Tamour Zubair) devolve a new wavelets algorithm to construct the numerical solution of nonlinear Bagley-Torvik equation of fractional order which will have less computational works, straight forward and better accuracy as compare to the existing technique. It is to be emphasized that proposed algorithm is tremendously simple but highly effective Moreover, this new pattern is proficient for reducing the computational work to a tangible level while still retaining a very high level of accuracy.

\section{Basic Definitions}

\section{Fractional Calculus [35]-[40]}

We give some basic definitions and properties of the fractional calculus theory which are used further in this paper.

Definition 1. A real function $f(t), t>0$ is said to be in the space $c_{\mu}, \mu \in R$ if there exists a real number $p(>\mu)$, such that $f(t)=t^{p} f_{1}(t)$, where $f_{1}(t) \in C[0, \infty]$, and it is said to be in the space $C_{\mu}^{m}$ iff $f^{m} \in C_{i}, m \in N$.

Definition 2. The Riemann-Liouville fractional integral operator of order $\alpha \geq 0$, of a function $f \in C_{\mu}, \mu \geq-1$, is defined as

$$
\begin{gathered}
J^{\alpha} f(t)=\frac{1}{\Gamma(\alpha)} \int_{0}^{t}(t-\tau)^{\alpha-1} f(\tau) \mathrm{d} \tau, \alpha>0, t>0, \\
J^{0} f(t)=f(t) .
\end{gathered}
$$

Properties of the operator $J^{\alpha}$ can be found in literature, we mention only the following: For $f \in C_{\mu}, \mu \geq-1, \alpha, \beta \geq 0$ and $\gamma>-1$ :

1) $J^{\alpha} J^{\beta} f(t)=J^{\alpha+\beta} f(t)$.

2) $J^{\alpha} J^{\beta} f(t)=J^{\beta} J^{\alpha} f(t)$.

3) $J^{\alpha} t^{\gamma}=\frac{\Gamma(\gamma+1)}{\Gamma(\alpha+\gamma+1)} t^{\alpha+\gamma}$.

The Riemann-Liouville derivative has certain drawbacks when trying to model real-world processes with fractional differential equations. Therefore, we shall introduce a improved fractional differential operator $D^{\alpha}$ proposed by $M$.

Definition 3. The fractional derivative of $f(t)$ in the Caputo sense is defined as

$$
D^{\alpha} f(t)=J^{m-\alpha} D^{m} f(t)=\frac{1}{\Gamma(m-\alpha)} \int_{0}^{t}(t-\tau)^{m-\alpha-1} f^{m}(t) \mathrm{d} t,
$$

for $m-1<\alpha \leq 1 m, m \in N, t>0, f \in C_{-1}^{m}$. For the Caputo's derivative we have 
$D^{\alpha} C=0, C$ is a constant,

$$
D^{\alpha} x^{k}= \begin{cases}0, & \text { for } n \in \mathbb{N}_{0} \text { and } n<\lceil\alpha\rceil ; \\ \frac{\Gamma(k+1)}{\Gamma(k+\alpha+1)} x^{k-\alpha}, & \text { for } n \in \mathbb{N}_{0} \text { and } n \geq\lceil\alpha\rceil .\end{cases}
$$

We use the ceiling function $\lceil\alpha\rceil$ to denote the smallest integer greater than or equal to $\alpha$, and $\mathbb{N}_{0}=\{0,1,2, \cdots\}$. Recall that for $\alpha \in \mathbb{N}$, the Caputo differential operator coincides with the usual differential operator of integer order.

\section{Bagley-Torvik Equations}

Bagley-Torvik equation assumes an extremely vital part to study the performance of different material by application of fractional calculus [40] [41]. It has increased its significance in many fields of industrial and applied sciences. Precisely, the equation with $1 / 2$ order derivative or $3 / 2$ order derivative can be model the frequency dependent damping materials. The summed up form of Bagley-Torvik equation is given

$$
\sigma \frac{\mathrm{d}^{\alpha} u(t)}{\mathrm{d} t^{\alpha}}+\omega \frac{\mathrm{d}^{\beta} u(t)}{\mathrm{d} t^{\beta}}+\mu(y(t))^{n}=f(t), \quad 0<t \leq \mathrm{T} .
$$

with initial condition

$$
\frac{\mathrm{d}^{p}}{\mathrm{~d} t^{p}} u(0)=l_{p}, \quad p=0,1
$$

with boundary condition at $t=t_{0}$, for $0<t_{0} \leq T$, is given by

$$
\frac{\mathrm{d}^{p}}{\mathrm{~d} t^{p}} u\left(t_{0}\right)=m_{p}, \quad p=0,1 \text {. }
$$

where $n$ is the nonlinear operator of the equation, $u(t)$ is unknown function. $\sigma, \omega$ and $\mu$ are the constant coefficients, $T$ is the constant representing the span of input in close interval $[0, \mathrm{~T}]$, and $l_{k}, m_{k}$ are contents. When we have

$$
n=1, \sigma=M, \mu=k, \omega=2 S \sqrt{\epsilon \rho}
$$

where $M$ is mass of the rigid plate, $k$ is stiffness of the spring, $S$ is the area of plate immersed in Newtonian fluid, $\epsilon$ is the velocity, and $\rho$ is the fluid density then equation (1) represents the motion of large thin plate in a Newtonian fluid [39]. Similarly, linearly damped fractional oscillator with the damping term has the fraction derivative $\beta=\frac{3}{2}$.

Further, we will discuss mathematical modeling of BT equation with feed-forward artificial neural network. The solution $u$ of the fractional differential equation along with its $v$ arbitrary order derivative $\frac{\mathrm{d}^{v} u}{\mathrm{~d} t^{v}}$ can be approximated by the following continuous mapping as a neural network methodology [41] [42] [43] [44]:

$$
\begin{gathered}
\tilde{u}(t)=\sum_{i=1}^{h} \gamma_{i} \vartheta\left(\aleph_{i} t+\chi_{i}\right), \\
\frac{\mathrm{d}^{v} \tilde{u}}{\mathrm{~d} t^{v}}=\sum_{i=1}^{h} \gamma_{i} \frac{\mathrm{d}^{v}}{\mathrm{~d} t^{v}} \vartheta\left(\aleph_{i} t+\chi_{i}\right),
\end{gathered}
$$


where $\gamma_{i}, \aleph_{i}$ and $\chi_{i}$ are bounded real valued adaptive parameters, $h$ is the number of neurons and $f$ is the active function taken as exponential function.

Fractional differential equation neural networks (FDN-NNs) can be approximate as

$$
\begin{gathered}
\tilde{u}(t)=\sum_{i=1}^{h} \gamma_{i} \mathrm{e}^{\left(\aleph_{i} t+\chi_{i}\right)}, \\
\frac{\mathrm{d}^{v} \tilde{u}}{\mathrm{~d} t^{\nu}}=\sum_{i=1}^{h} \gamma_{i} \aleph_{i}^{N} \mathrm{e}^{\left(\aleph_{i} t+\chi_{i}\right)},
\end{gathered}
$$

for $v=2$, we get

$$
\frac{\mathrm{d}^{2} \tilde{u}}{\mathrm{~d} t^{2}}=\sum_{i=1}^{h} \gamma_{i} \aleph_{i}^{2} \mathrm{e}^{\left(\aleph_{i} t+\chi_{i}\right)},
$$

Using Definition 4 , for $v=\frac{3}{2}$, we get

$$
\frac{\mathrm{d}^{\frac{3}{2}} \tilde{u}}{\mathrm{~d} t^{\frac{3}{2}}}=\sum_{i=1}^{h} \gamma_{i} \mathrm{e}^{\left(\gamma_{i}\right)} t^{-\frac{3}{2}} E_{1,-\frac{1}{2}}\left(\aleph_{i} t\right) .
$$
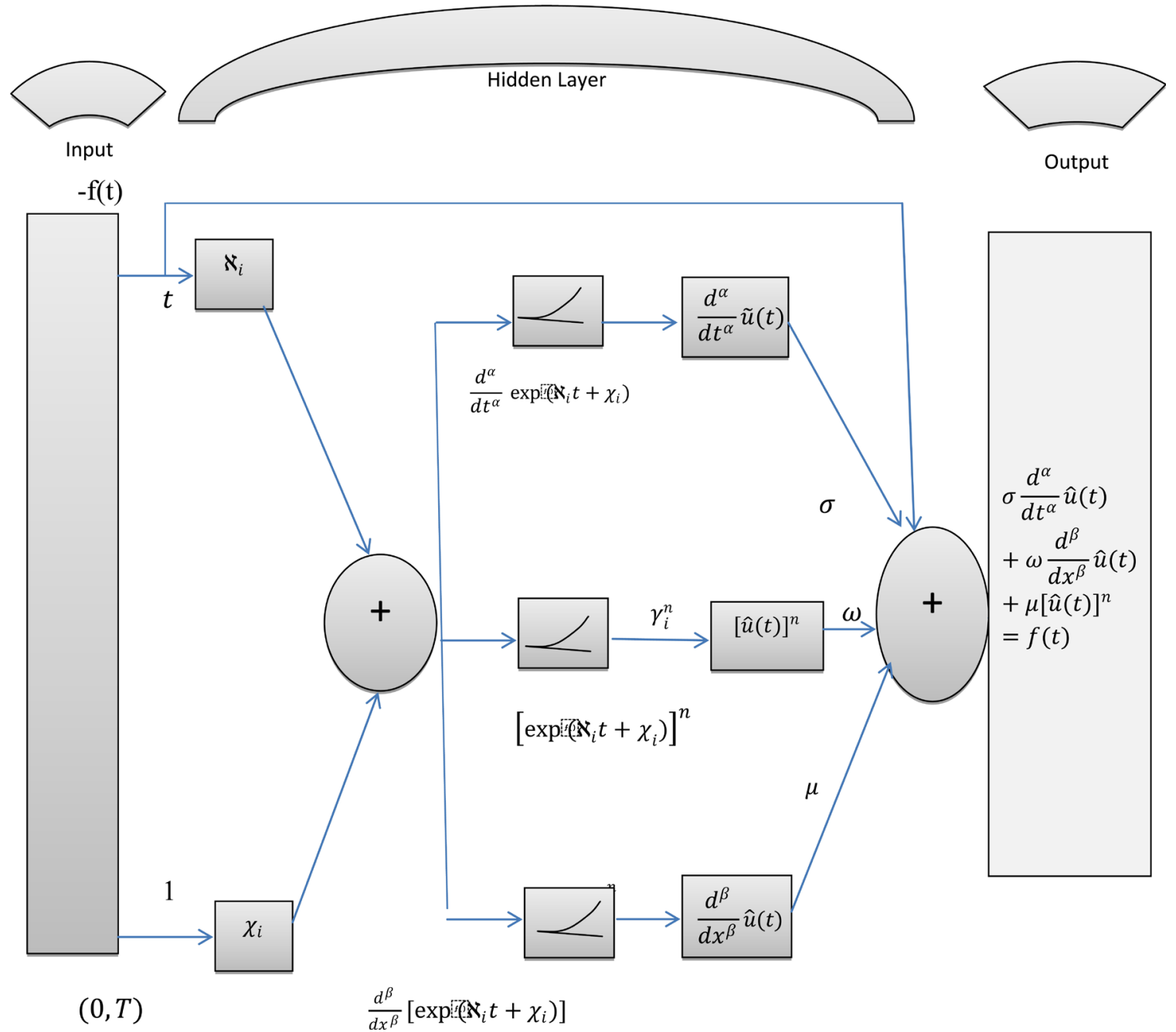

Figure 1. FDE-NN architecture of Bagley-Torvik equation. 
The mathematical model can be the linear combinations of the networks represented above. The FDE-NN architecture formulated for Bagley-Torvik equation can be seen in Figure 1. It is clear that the solution $u$ can be approximated with $\tilde{u}$ subject to finding appropriate unknown weights.

\section{Hermite Polynomials [45]}

It is classical orthogonal polynomials play very important role in probability. It has wide applications in numerical analysis as finite element methods as shape functions for beams. They are also applicable in physical quantum theory. Hermite polynomials are categorized into two kinds

The Probabilists Hermite polynomials are the solutions of

$$
\left(\mathrm{e}^{\varnothing} y^{\prime}\right)^{\prime}+\lambda\left(\mathrm{e}^{\varnothing} y\right)=0, \varnothing<0, \lambda>0 .
$$

where $\varnothing=\frac{x^{2}}{2}$ and $\lambda$ is a constant, with the boundary conditions that $y$ should be polynomially bounded at infinity. The above equation can be written in the form of eigen value problem

$$
L[y]=y^{\prime \prime}+x y^{\prime}=-\lambda y,
$$

solutions are the Eigen functions of the differential operator $L$. This equation is called Hermite equation, although the term is also used for the closely related equation

$$
y^{\prime \prime}-2 x y^{\prime}=2 \lambda y .
$$

whose solutions are the Physicists Hermites Polynomials, which is the second kind of Hermite polynomials.

The Hermite polynomials is given by

$$
H_{n}(x)=(-1)^{n} \mathrm{e}^{\varnothing_{1}} \frac{\mathrm{d}^{n}}{\mathrm{~d} x^{n}} \mathrm{e}^{\varnothing_{2}}, \varnothing_{1}>0, \varnothing_{2}<0 .
$$

where $\varnothing_{1}= \begin{cases}x^{2}, & \text { for Physicists Hermites Polynomials, } \\ \frac{x^{2}}{2}, & \text { for Probabilists Hermites Polynomials. }\end{cases}$

and also $\varnothing_{1}=-\varnothing_{2}$.

\begin{tabular}{cc}
\hline Probabilists Hermites Polynomials & Physicists Hermites Polynomials \\
\hline$H e_{0}=1$, & $H_{0}=1$, \\
$H e_{1}=x$, & $H_{1}=2 x$, \\
$H e_{2}=x^{2}-1$, & $H_{2}=4 x^{2}-2$, \\
$H e_{3}=x^{3}-3 x$, & $H_{3}=8 x^{3}-12 x$, \\
$\mathrm{He}_{4}=x^{4}-6 x^{2}+3$, & $H_{4}=x^{4}-6 x^{2}+3$, \\
$\vdots$ & $\vdots$ \\
\hline
\end{tabular}


$H_{n}(x)$ and $H_{n}(x)$ the two branches of Hermite polynomial of degree $n$, which are orthogonal with respect to weigh function.

$$
w(x)= \begin{cases}\mathrm{e}^{\frac{\varnothing_{1}}{2}}, & \text { for Physicists Hermites Polynomials, } \\ \mathrm{e}^{2 \varnothing_{1}}, & \text { for Probabilists Hermites Polynomials. }\end{cases}
$$

Here we have $\varnothing_{1}<0$.

Further we have orthogonality $R(x)$ is given by

$$
R(x)= \begin{cases}\int_{-\infty}^{\infty} H e_{m}(x) H e_{n}(x) w(x) \mathrm{d} x=\sqrt{2 \pi} n ! \delta_{n m}, & \text { for Probabilists, } \\ \int_{-\infty}^{\infty} H_{m}(x) H_{n}(x) w(x) \mathrm{d} x=\sqrt{\pi} 2^{n} n ! \delta_{n m}, & \text { for Physicists. }\end{cases}
$$

A function $u(x) \in L_{w(x)}^{2}(\infty,-\infty)$ can be express in term of Hermite polynomials

$$
u(x)=\sum_{i=0}^{\infty} a_{i} H_{i}(x) .
$$

where $a_{i}$ coefficients is given by

$$
a_{i}=\frac{1}{\varrho_{i}} \int_{-\infty}^{\infty} u(x) H_{i}(x) w(x) \mathrm{d} x .
$$

where $\varrho_{i}=\sqrt{\pi} 2^{n} n$ !.

\section{Fractional Form of Hermite Polynomials [35]-[40]}

The explicit formula of Hermites polynomials is

$$
P_{n}(x)=n ! \sum_{m=0}^{\left\lfloor\frac{n}{2}\right\rfloor}\left\{\frac{(-1)^{m}}{m !(n-m) !} X^{\prime}\right\} \text {. }
$$

where $X^{\prime}$ is given by

$$
X^{\prime}= \begin{cases}(2 x)^{n-2 m}, & \text { for Physicists Hermites Polynomials, } \\ \frac{x^{n-2 m}}{2^{m}}, & \text { for Probabilists Hermites Polynomials. }\end{cases}
$$

Further we have

$$
P_{n}(x)=n ! \sum_{m=0}^{\left\lfloor\frac{n}{2}\right\rfloor}\left\{\delta_{m} X^{\prime}\right\}
$$

where $\delta_{m}$ is given by

$$
\delta_{m}=\frac{(-1)^{m}}{m !(n-m) !}
$$

A function $u(x) \in L_{w(x)}^{2}(\infty,-\infty)$ can be express in term of Hermite polynomials

$$
\tilde{u}(x)=\sum_{n=0}^{M} a_{k} P_{n}(x) .
$$

where $P(x)$ are Hermites polynomials. Using $\left(1^{\star}\right)-(3)$ and definition of fractional derivative, we get the following

$$
D^{\alpha} \tilde{u}(x) \cong \sum_{n=\alpha}^{M}\left(n ! \sum_{m=0}^{\xi} a_{n} \delta_{m} \chi_{n m}^{\alpha} \aleph^{\prime}\left(X^{\prime}\right)^{-\alpha}\right) .
$$


where $\aleph^{\prime}, \chi_{n m}^{\alpha}$ and $\xi$ is given by $\aleph^{\prime}=2^{\alpha}, \chi_{n m}^{\alpha}=\frac{\Gamma(n-2 m+1)}{\Gamma(n-2 m-\alpha+1)}$ and $\xi=\frac{n-\alpha}{2}$.

Note that only for $\aleph^{\prime \prime}$, we have following

$$
\aleph^{\prime}= \begin{cases}\alpha>0, & \text { for Physicists Hermites Polynomials, } \\ \alpha<0, & \text { for Probabilists Hermites Polynomials. }\end{cases}
$$

\section{a) Methodology}

Consider the multi order fractional differential equation (1) as

$$
\begin{gathered}
D^{\alpha} u(x)=\varphi\left(x ; u(x), D^{\beta_{1}} u(x), D^{\beta_{2}} u(x), \cdots, D^{\beta_{n}} u(x)\right), \\
u^{(n)}(0)=u_{n}, n=0,1,2, \cdots, m-1,
\end{gathered}
$$

where $u(x)$ is the unknown function, to be determined. The proposed technique for solving Equation (5) proceeds in the following three steps:

Step 1: According to the proposed algorithm we assume the following trial solution

$$
\tilde{u}(x)=\sum_{k=0}^{M} a_{k} P_{k}(x)=\boldsymbol{U}^{\mathrm{T}} \boldsymbol{P}(x),
$$

where $\boldsymbol{U}=\left[a_{0}, a_{1}, a_{2}, \cdots\right]^{\mathrm{T}}$ and $\boldsymbol{P}(x)=\left[P_{0}(x), P_{1}(x), P_{2}(x), \cdots\right]^{\mathrm{T}}$.

where $P_{k}(x)$ are Hermite polynomials of degree $k$ defined in Equation (6) and $a_{k}$ are unknown parameters, to be determined.

Step 2: Substituting Equation (6) into Equation (5), we get

$$
\begin{gathered}
\boldsymbol{U}^{\mathrm{T}} D^{\alpha} \boldsymbol{P}(x)=\varphi\left(x ; \boldsymbol{U}^{\mathrm{T}} \boldsymbol{P}(x), \boldsymbol{U}^{\mathrm{T}} D^{\beta_{1}} \boldsymbol{P}(x), \boldsymbol{U}^{\mathrm{T}} D^{\beta_{2}} \boldsymbol{P}(x), \cdots, \boldsymbol{U}^{\mathrm{T}} D^{\beta_{n}} \boldsymbol{P}(x)\right), \beta_{1} \\
\boldsymbol{U}^{\mathrm{T}} \boldsymbol{P}(x)(0)=u_{n},
\end{gathered}
$$

Using (4) we have

$$
\begin{gathered}
\sum_{n=\{\alpha\rceil}^{h}\left(n ! \sum_{m=0}^{\xi} a_{n} \delta_{m} \chi_{n m}^{\alpha} \aleph^{\prime}\left(X^{\prime}\right)^{-\alpha}\right) \\
=\varphi\left(x ; \sum_{k=0}^{M} a_{k} P_{k}(x), \sum_{n=\left\lceil\beta_{1}\right]}^{M}\left(n ! \sum_{m=0}^{\xi} a_{n} \delta_{m} \chi_{n m}^{\beta_{1}} \aleph^{\prime}\left(X^{\prime}\right)^{-\beta_{1}}\right),\right. \\
\sum_{n=\left\{\beta_{2}\right]}^{M}\left(n ! \sum_{m=0}^{\xi} a_{n} \delta_{m} \chi_{n m}^{\beta_{2}} \aleph^{\prime}\left(X^{\prime}\right)^{-\beta_{2}}\right), \cdots, \\
\left.\sum_{n=\left\{\beta_{n}\right]}^{M}\left(n ! \sum_{m=0}^{\xi} a_{n} \delta_{m} \chi_{n m}^{\beta_{n}} \aleph^{\prime}\left(X^{\prime}\right)^{-\beta_{n}}\right)\right), \\
\boldsymbol{U}^{\mathrm{T}} \boldsymbol{P}(x)(0)=u_{n} .
\end{gathered}
$$

Step 3: Further we Assume suitable collocation point for Equation (7). Therefore, we obtained system has $M+1$ equations and $M+1$ unknowns. Solving this system gives the unknown coefficients using Conjugate Gradient Method. Putting these constant into trial solution, we can obtained the approximate/exact solutions of linear/nonlinear fractional differential Equation (5).

b) Approximation by Hermite Polynomials [45]

Let us define $\Omega=\{x,-\infty<x<\infty\}$ and $\gamma_{N}=\operatorname{span}\left\{P_{0}(x), P_{1}(x), \cdots, P_{N}(x)\right\}$. The $L_{w(x)}^{2}(\Omega)$-orthogonal projection $\pi_{N}: L^{2}(\Omega) \rightarrow \gamma_{N}$ be the mapping and we 
have

$$
\left\langle\pi_{N}(z)-z\right\rangle=0, \forall \theta \in \gamma_{N}
$$

Due to the orthogonality property, we can write it as

$$
\pi_{N}(z)=\sum_{k=0}^{N-1} a_{k} P_{k}(x),
$$

where $a_{i}(i=0,1, \cdots, N-1)$ are the constants in the following form

$$
a_{i}=\frac{1}{\varrho_{i}}\left\langle u(x), P_{k}\right\rangle L_{w(x)}^{2}
$$

\section{Numerical Simulation}

In this section, we apply new algorithm to construct approximate/exact solutions fractional differential equation. Numerical results are very encouraging.

Case 1 In Equation (1), we take $\alpha=2, \beta=\frac{3}{2}, \sigma=\omega=\mu=n=1$, $f(t)=2+4 \sqrt{\frac{t}{\pi}}+t^{2}, l_{p}=0, t_{0}=1, m_{p}=1,2$. The close form solution is $t^{2}$.

Consider the trial solutions for $M=2$ as

$$
\tilde{u}(t)=\sum_{n=0}^{M} a_{k} P_{n}(t) .
$$

Using the trail solution into Equation (1) and proceed it according to Step 1 and Step 2, then we collocate it further to generate the system of equations. Solve the system of equations along with initial conditions, we get the values of constants

\begin{tabular}{cccc}
\hline Hermite's & $a_{0}$ & $a_{1}$ & $a_{2}$ \\
Physicists & $\frac{1}{2}$ & 0 & $\frac{1}{4}$ \\
Probabilists & 1 & 0 & 1 \\
\hline
\end{tabular}

Finally, we get the approximate solution

$$
u(t)=t^{2} .
$$

which is exact solution.

Case 2 In Equation (1), we take $\alpha=2, \beta=\frac{3}{2}, \sigma=\omega=\mu=n=1$, $f(t)=2+4 \sqrt{\frac{t}{\pi}}+t^{2}, l_{0}=0, t_{0}=5, m_{0}=25$. The close form solution is $t^{2}$.

Consider the trial solutions for $M=2$ as

$$
\tilde{u}(t)=\sum_{n=0}^{M} a_{k} P_{n}(t) .
$$

Using the trail solution into Equation (1) and proceed it according to Step 1 and Step 2, then we collocate it further to generate the system of equations. Solve the system of equations along with initial conditions, we get the values of constants 


\begin{tabular}{cccc}
\hline Hermite's & $a_{0}$ & $a_{1}$ & $a_{2}$ \\
Physicists & $\frac{1}{2}$ & 0 & $\frac{1}{4}$ \\
Probabilists & 1 & 0 & 1 \\
\hline
\end{tabular}

Finally, we get the approximate solution

$$
u(t)=t^{2} .
$$

which is exact solution.

Case 3 In Equation (1), we take $\alpha=2, \beta=\frac{3}{2}, \sigma=\omega=\mu=n=1$, $f(t)=t+1, l_{p}=1$. The close form solution is $1+t$.

This equation can be simplify by using

$$
U(t)=u(t)-1-t .
$$

Consider the trial solutions for $M=2$ as

$$
\tilde{u}(t)=\sum_{n=0}^{M} a_{k} P_{n}(t) .
$$

Using the trail solution into Equation (1) and proceed it according to Step 1 and Step 2, then we collocate it further to generate the system of equations. Solve the system of equations along with initial conditions, we get the values of constants

\begin{tabular}{cccc}
\hline Hermite's & $a_{0}$ & $a_{1}$ & $a_{2}$ \\
Physicists & 1 & $\frac{1}{2}$ & 0 \\
Probabilists & 1 & 1 & 0 \\
\hline
\end{tabular}

Finally, we get the approximate solution

$$
u(t)=1+t .
$$

which is exact solution.

Case 4 In Equation (1), we take $\alpha=2, \sigma=n=\mu=1, \omega=\frac{1}{2}$, $f(t)=3+t^{2}\left(\frac{t^{-\beta}}{\Gamma(3-\beta)}+1\right), \quad p=0, l_{0}=1, t_{0}=1, \quad m_{0}=2$. The close form solution is $1+t^{2}$.

Using the trail solution into Equation (1) and proceed it according to Step 1 and Step 2, then we collocate it further to generate the system of equations. Solve the system of equations along with initial conditions, we get the values of constants

\begin{tabular}{cccc}
\hline Hermite's & $a_{0}$ & $a_{1}$ & $a_{2}$ \\
Physicists & $\frac{3}{2}$ & 0 & $\frac{1}{4}$ \\
Probabilists & 2 & 0 & 1 \\
\hline
\end{tabular}


Finally, we get the approximate solution

$$
u(t)=1+t^{2} .
$$

which is exact solution.

Case 5. In Equation (1), we take $\alpha=2, \sigma=n=\mu=1, \omega=\frac{1}{2}$,

$f(t)=4 t^{2}(5 t-3)+\omega t^{4-\beta}\left(\frac{120}{\Gamma(6-\beta)} t-\frac{24}{\Gamma(5-\beta)}\right)+\mu t^{4}(t-1), \quad p=0, \quad l_{0}=0$, $t_{0}=1, m_{0}=0$. The close form solution is $t^{4}(t-1)$.

The numerical solution is represented in Table 1 in case of $M=2,5$ and $\beta=0.3$, while the error for various values of $\beta=0$ and $\beta=0.5$ are represented in Table 2. There is a graphical comparison between exact and approximate solution represented in Figure 2.

Table 1. Numerical comparison between exact and approximate solution for deferent values of $M$.

\begin{tabular}{ccc}
\hline$x$ & $M=2$ & $M=5$ \\
\hline 0 & $0.00000 \mathrm{E}+00$ & $0.00000 \mathrm{E}+00$ \\
0.1 & $9.00000 \mathrm{E}-05$ & $6.42250 \mathrm{E}-45$ \\
0.2 & $1.28000 \mathrm{E}-03$ & $9.13422 \mathrm{E}-44$ \\
0.3 & $5.67000 \mathrm{E}-03$ & $4.04617 \mathrm{E}-43$ \\
0.4 & $1.53600 \mathrm{E}-02$ & $1.09611 \mathrm{E}-42$ \\
0.5 & $3.12500 \mathrm{E}-02$ & $2.23003 \mathrm{E}-42$ \\
0.6 & $5.18400 \mathrm{E}-02$ & $3.69936 \mathrm{E}-42$ \\
0.7 & $7.20300 \mathrm{E}-02$ & $5.14014 \mathrm{E}-42$ \\
0.8 & $8.19200 \mathrm{E}-02$ & $5.84590 \mathrm{E}-42$ \\
0.9 & $6.56100 \mathrm{E}-02$ & $4.68200 \mathrm{E}-42$ \\
1.0 & $0.00000 \mathrm{E}+00$ & $0.00000 \mathrm{E}+00$ \\
\hline
\end{tabular}

Error $=\mid$ Exact Solution - Approximate solution $\mid$.

Table 2. Numerical comparison between exact approximate solutions for different values of $\beta$.

\begin{tabular}{ccc}
\hline$x$ & $\beta=0$ & $\beta=0.5$ \\
\hline 0 & $0.00000 \mathrm{E}+00$ & $4.00000 \mathrm{E}-100$ \\
0.1 & $9.08224 \mathrm{E}-32$ & $7.57685 \mathrm{E}-45$ \\
0.2 & $1.80074 \mathrm{E}-31$ & $1.07760 \mathrm{E}-43$ \\
0.3 & $2.65365 \mathrm{E}-31$ & $4.77341 \mathrm{E}-43$ \\
0.4 & $3.42665 \mathrm{E}-31$ & $1.29312 \mathrm{E}-42$ \\
0.5 & $4.05488 \mathrm{E}-31$ & $2.63085 \mathrm{E}-42$ \\
0.6 & $4.44066 \mathrm{E}-31$ & $4.36426 \mathrm{E}-42$ \\
0.7 & $4.44538 \mathrm{E}-31$ & $6.06400 \mathrm{E}-42$ \\
0.8 & $3.88121 \mathrm{E}-31$ & $6.89662 \mathrm{E}-42$ \\
0.9 & $2.50300 \mathrm{E}-31$ & $5.52352 \mathrm{E}-42$ \\
1.0 & $8.00000 \mathrm{E}-100$ & $2.00000 \mathrm{E}-99$
\end{tabular}

Error $=\mid$ Exact Solution - Approximate solution $\mid$. 


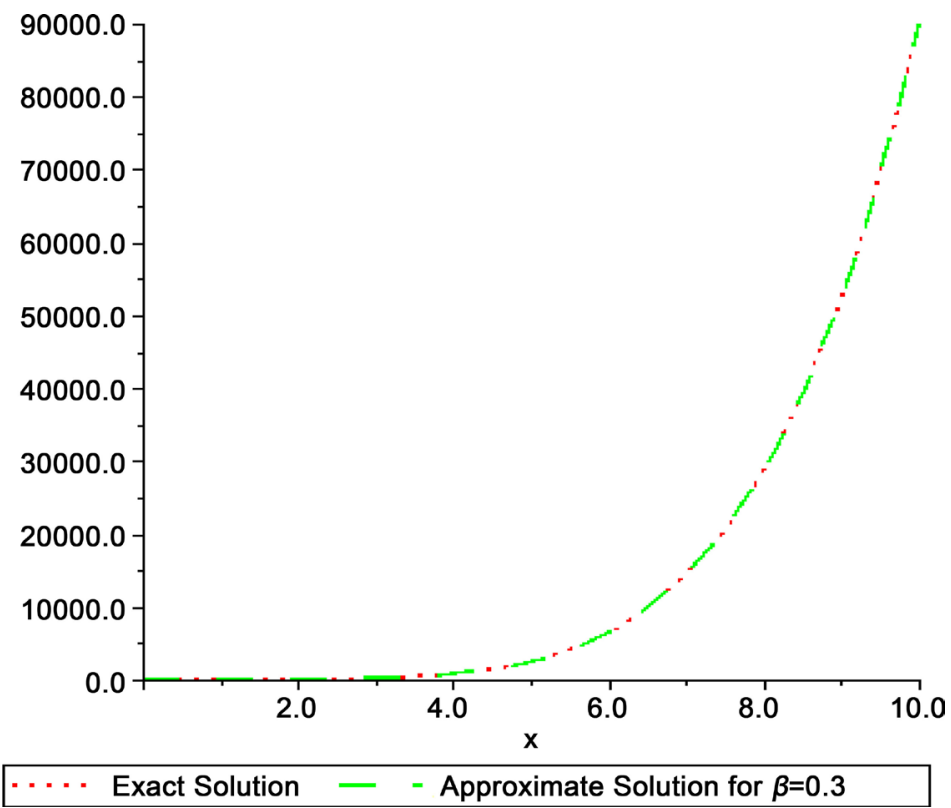

Figure 2. Graphysical comparision between exact and approximted solution.

\section{Conclusions}

All the facts and findings of the paper are summarized as follow:

- This paper provides novel study of Bagley-Torvik equations of fractional order in different situations by using newly suggested Hermite Polynomial scheme.

- Implementation of this methodology is moderately relaxed and with the help of this suggested algorithm, complicated problems can be tackled.

- It is to be highlighted that the suggested comparison gives attentive respond regarding some particular issues for values of $M$, which demonstrates viability of the proposed framework. Likewise, the reliability of the application provided this technique a more comprehensive suitability.

\section{References}

[1] Deng, W. (2008) Finite Element Method for the Space and Time Fractional Fokker-Planck Equation. SIAM Journal on Numerical Analysis, 47, 204-226. http://epubs.siam.org/doi/abs/10.1137/080714130 https://doi.org/10.1137/080714130

[2] Gao, G.H., Sun, Z.Z. and Zhang, Y.N. (2012) A Finite Difference Scheme for Fractional Sub-Diffusion Equations on an Unbounded Domain Using Artificial Boundary Conditions. Journal of Computational Physics, 231, 2865-2879. https://www.hindawi.com/journals/mpe/2012/924956/ https://doi.org/10.1016/j.jcp.2011.12.028

[3] Momani, S., Odibat, Z. and Erturk, V.S. (2007) Generalized Differential Transform Method for Solving a Space- and Time-Fractional Diffusion-Wave Equation. Physics Letters A, 370, 379-387.

https://www.mutah.edu.jo/userhomepages/shmomani/public.htm https://doi.org/10.1016/j.physleta.2007.05.083

[4] Odibat, Z. and Momani, S. (2008) A Generalized Differential Transform Method for 
Linear Partial Differential Equations of Fractional Order. Applied Mathematics Letters, 21, 194-199.

https://pdfs.semanticscholar.org/7250/38695674846cdbb7c5f5d2d9cbcb5f4e0fc9.pdf https://doi.org/10.1016/j.aml.2007.02.022

[5] Hu, Y., Luo, Y. and Lu, Z. (2008) Analytical Solution of the Linear Fractional Differential Equation by Adomian Decomposition Method. Journal of Computational and Applied Mathematics, 215, 220-229.

https://pdfs.semanticscholar.org/58a6/966afe30ccc2f7bbc6b17319d6a3d1a663ba.pdf https://doi.org/10.1016/j.cam.2007.04.005

[6] El-Sayed, A.M.A. and Gaber, M. (2006) The Adomian Decomposition Method for Solving Partial Differential Equations of Fractal Order in Finite Domains. Physics Letters A, 359, 175-182.

https://www.researchgate.net/publication/223114291_The_Adomian_decompositio $\underline{\text { n_method_for_solving_partial_differential_equations_of_fractal_order_in_finite_d }}$ omains https://doi.org/10.1016/j.physleta.2006.06.024

[7] El-Sayed, A.M.A., Behiry, S.H. and Raslan, W.E. (2010) Adomian's Decomposition Method for Solving an Intermediate Fractional Advection-Dispersion Equation. Computers \& Mathematics with Applications, 59, 1759-1765.

http://www.sciencedirect.com/science/article/pii/S0898122109005537 https://doi.org/10.1016/j.camwa.2009.08.065

[8] Inc, M. (2008) The Approximate and Exact Solutions of the Space- and Time-Fractional Burgers Equations with Initial Conditions by Variational Iteration Method. Journal of Mathematical Analysis and Applications, 345, 476-484.

http://www.sciencedirect.com/science/article/pii/S0022247X08003739 https://doi.org/10.1016/j.jmaa.2008.04.007

[9] Odibat, Z. and Momani, S. (2009) The Variational Iteration Method: An Efficient Scheme for Handling Fractional Partial Differential Equations in Fluid Mechanics. Computers \& Mathematics with Applications, 58, 2199-2208.

http://www.sciencedirect.com/science/article/pii/S0898122109001436 https://doi.org/10.1016/j.camwa.2009.03.009

[10] Wu, G.C. and Lee, E.W.M. (2010) Fractional Variational Iteration Method and Its Application. Physics Letters A, 374, 2506-2509.

https://zulfahmed.files.wordpress.com/2015/06/2010-physics-letters-section-a-gene ral-atomic-and-solid-state-physics-37425.pdf https://doi.org/10.1016/j.physleta.2010.04.034

[11] He, J.H. (2003) Homotopy Perturbation Method: A New Nonlinear Analytical Technique. Applied Mathematics and Computation, 135, 73-79.

https://www.researchgate.net/publication/242791050_Homotopy_perturbation_met hod_A_new_nonlinear_analytical_technique https://doi.org/10.1016/S0096-3003(01)00312-5

[12] Zubair, T., Hamid, M., Saleem, M. and Mohyud-Din, S.T. (2015) Numerical Solution of Infinite Boundary Integral Equations. International Journal of Modern Applied Physics, 5, 18-25.

https://www.researchgate.net/publication/311202189_Numerical_Solution_of_Infin ite_Boundary_Integral_Equations

[13] Bin, Z. (2012) ( $\left.G^{\prime} / G\right)$-Expansion Method for Solving Fractional Partial Differential Equations in the Theory of Mathematical Physics. Communications in Theoretical Physics, 58, 623-630.

http://ctp.itp.ac.cn/EN/article/downloadArticleFile.do?attachType=PDF\&id=15873 
https://doi.org/10.1088/0253-6102/58/5/02

[14] Usman, M. and Mohyud-Din, S.T. (2013) Traveling Wave Solutions of $7^{\text {th }}$ Order Kaup Kuperschmidt and Lax Equations of Fractional-Order. International Journal of Advances in Applied Mathematics and Mechanics, 1, 17-34. http://www.ijaamm.com/uploads/2/1/4/8/21481830/paper_2_sayed.pdf

[15] Usman, M. and Mohyud-Din, S.T. (2014) $U$-Expansion Method for 5th Order Kaup Kuperschmidt and Lax Equation of Fractional Order. International Journal of Modern Mathematical Sciences, 9, 63-81.

http://www.modernscientificpress.com/journals/ViewArticle.aspx?XBq7Uu+HD/8e RjFUGMqlRUFMGJSojFCd8JeYyjsMmViTRlEyTDVgYMCmavqTokhF

[16] Alzaidy, J.F. (2013) Fractional Sub-Equation Method and Its Applications to the Space-Time Fractional Differential Equations in Mathematical Physics. British Journal of Mathematics \& Computer Science, 3, 153-163.

https://www.researchgate.net/publication/271263179_Fractional_Sub-Equation_Me thod_and_its_Applications_to_the_Space-Time_Fractional_Differential_Equations in_Mathematical_Physics https://doi.org/10.9734/BJMCS/2013/2908

[17] Alzaidy, J.F. (2013) The Fractional Sub-Equation Method and Exact Analytical Solutions for Some Nonlinear Fractional PDEs. American Journal of Mathematical Analysis, 1, 14-19. http://pubs.sciepub.com/ajma/1/1/3/

[18] Jafari, H., Yousefi, S.A., Firoozjaee, M.A., Momani, S. and Khalique, C.M. (2011) Application of Legendre Wavelets for Solving Fractional Differential Equations. Computers \& Mathematics with Applications, 62, 1038-1045. http://www.sciencedirect.com/science/article/pii/S0898122111003257 https://doi.org/10.1016/j.camwa.2011.04.024

[19] Khader, M.M., El Danaf, T.S. and Hendy, A.S. (2013) A Computational Matrix Method for Solving Systems of High Order Fractional Differential Equations. Applied Mathematical Modelling, 37, 4035-4050.

https://www.researchgate.net/publication/235916821_A_computational_matrix_me thod_for_solving_systems_of_high_order_fractional_differential_equations https://doi.org/10.1016/j.apm.2012.08.009

[20] Zhu, L. and Fan, Q.B. (2012) Solving Fractional Nonlinear Fredholm Integro-Differential Equations by the Second Kind Chebyshev Wavelet. Communications in Nonlinear Science and Numerical Simulation, 17, 2333-2341.

https://advancesindifferenceequations.springeropen.com.previewlive.oscarjournals.sprin ger.com/track/pdf/10.1186/s13662-017-10856?site=advancesindifferenceequations.sprin geropen.com.previewlive.oscarjournals.springer.com https://doi.org/10.1016/j.cnsns.2011.10.014

[21] Agarwal, N. (1953) A Propos d'unc Note de H4. Pierre Humbert, C. R. Se'ances Acad. Sci., 236, 2031-2032. http://jnus.org/pdf/1/2014/1/1038.pdf

[22] Li, Y. and Zhao, W. (2010) Haar Wavelets Operational Matrix of Fractional Order Integration and Its Applications in Solving the Fractional Order Differential Equations. Applied Mathematics and Computation, 216, 2276-2285.

http://dl.acm.org/citation.cfm?id=2641414 https://doi.org/10.1016/j.amc.2010.03.063

[23] Khader, M.M. and Hendy, A.S. (2012) The Approximate and Exact Solutions of the Fractional-Order Delay Differential Equations Using Legendre Pseudo Spectral Method. International Journal of Pure and Applied Mathematics, 74, 287-297. http://www.ijpam.eu/contents/2012-74-3/1/1.pdf

[24] Sweilam, N.H. and Khader, M.M. (2010) A Chebyshev Pseudo-Spectral Method for 
Solving Fractional Integro-Differential Equations. The ANZIAM Journal, 51, 464475.

https://www.cambridge.org/core/journals/anziam-journal/article/div-classtitlea-cheby shev-pseudo-spectral-method-for-solving-fractional-order-integro-differential-equati onsdiv/D54540C52DE837C74F79EDB24A32FE71 https://doi.org/10.1017/S1446181110000830

[25] Beheshti, S., Khosravian-Arab, H. and Zare, I. (2012) Numerical Solution of Fractional Differential Equations by Using the Jacobi Polynomials. Bulletin of the Iran Mathematical Society, 39, 6461-6470.

http://bims.iranjournals.ir/article_947_b3ed3a5b2e22cf9386624a6699f3ff0d.pdf

[26] Xu, C.-L. and Guo, B.-Y. (2002) Laguerre Pseudospectral Method for Non-Linear Partial Differential Equations. Journal of Computational Mathematics-International Edition, 20, 413-428.

http://dergi.cumhuriyet.edu.tr/cumuscij/article/view/5000118841

[27] Razzaghi, M. and Yousefi, S. (2001) Legendre Wavelets Method for the Solution of Nonlinear Problems in the Calculus of Variations. Mathematical and Computing Modelling, 34, 45-54.

http://www.sciencedirect.com/science/article/pii/S0895717701000486

[28] Khader, M.M. (2012) Introducing an Efficient Modification of the Homotopy Perturbation Method by Using Chebyshev Polynomials. Arab Journal of Mathematical Sciences, 18, 61-71.

http://www.sciencedirect.com/science/article/pii/S131951661100051X https://doi.org/10.1016/j.ajmsc.2011.09.001

[29] Khader, M.M., Sweilam, N.H. and Mahdy, A.M.S. (2011) An Efficient Numerical Method for Solving the Fractional Diffusion Equation. Journal of Applied Mathematics and Bioinformatics, 1, 1-12. http://www.scienpress.com/upload/JAMB/Vol\%201_2_1.pdf

[30] Hussaini, M.Y. and Zang, T.A. (1987) Spectral Methods in Fluid Dynamics. Annual Review of Fluid Mechanics, 19, 339-367. http://www.annualreviews.org/doi/abs/10.1146/annurev.fl.19.010187.002011 https://doi.org/10.1146/annurev.fl.19.010187.002011

[31] Funaro, D. (1992) Polynomial Approximation of Differential Equations. Springer Verlag, New York, 8. http://www.springer.com/gb/book/9783662138786

[32] Khader, M.M., El Danaf, T.S. and Hendy, A.S. (2012) Efficient Spectral Collocation Method for Solving Multi-Term Fractional Differential Equations Based on the Generalized Laguerre Polynomials. Fractional Calculus Application, 3, 1-14. http://naturalspublishing.net/files/published/vk2130111wp11o.pdf

[33] Khader, M.M. (2011) On the Numerical Solutions for the Fractional Diffusion Equation. Communications in Nonlinear Science and Numerical Simulations, 16, 2535 2542. http://www.ijpam.eu/contents/2013-84-4/1/1.pdf

[34] Doha, E.H., Bahrawy, A.H. and Ezz-Eldien, S.S. (2011) Efficient Chebyshev Spectral Methods for Solving Multi-Term Fractional Orders Differential Equations. Applied Mathematical Modelling, 35, 5662-5672.

http://naturalspublishing.net/files/published/vk2130111wp11o.pdf https://doi.org/10.1016/j.apm.2011.05.011

[35] Dalir, M. and Bashour, M. (2010) Applications of Fractional Calculus. Applied Mathematical Sciences, 4, 1021-1032. https://pdfs.semanticscholar.org/b9f3/cebf62c66c7bc06eab009aa1d60d70a19312.pdf

[36] Schneider, K., Kevlahan, N.K.R. and Farge, M. (1997) Comparison of an Adaptive 
Wavelet Method and Nonlinearly Filtered Pseudospectral Methods for Two-Dimensional Turbulence. Theoretical and Computational Fluid Dynamics, 9, 919-206. https://link.springer.com/article/10.1007/s001620050040

[37] Erdelyi, A. (1955) Higher Transcendental Functions, McGrawHill, New York. http://apps.nrbook.com/bateman/Vol3.pdf

[38] Bagley, R.L. and Torvik, P.J. (1983) Fractional Calculus-A Different Approach to the Analysis of Viscoelastically Damped Structures. AIAA Journal, 21, 741-748. https://arc.aiaa.org/doi/abs/10.2514/3.8142 https://doi.org/10.2514/3.8142

[39] Podlubny, I. (1999) Fractional Differential Equations, vol. 198 of Mathematics in Science and Engineering, Academic Press, San Diego. http://www.sciepub.com/reference/3051

[40] Bagley, R.L. and Torvik, P.J. (1984) On the Appearance of the Fractional Derivative in the Behavior of Real Materials. Journal of Applied Mechanics, 51, 294-298.

http://appliedmechanics.asmedigitalcollection.asme.org/article.aspx?articleid $=1407$ $\underline{517}$ https://doi.org/10.1115/1.3167615

[41] Zahoor, M.A., Khan Raja, J.A. and Qureshi, I.M. (2010) Heuristic Computational Approach Using Swarm Intelligence in Solving Fractional Differential Equations. Proceedings of the 12 th Annual Genetic and Evolutionary Computation Conference, Portland, 2023-2026.

https://www.hindawi.com/journals/cin/2012/721867/ref/

[42] Ray, S.S. and Bera, R.K. (2005) Analytical Solution of the Bagley Torvik Equation by Adomian Decomposition Method. Applied Mathematics and Computation, 168, 398-410. http://www.ijpam.eu/contents/2016-110-2/3/

[43] Zahoor, R.M.A., Khan, J.A. and Qureshi, I.M. (2009) Evolutionary Computation Technique for Solving Riccati Differential Equation of Arbitrary Order. World Academy of Science, Engineering and Technology, 58, 531-536.

https://scholar.google.com.pk/citations?view_op=view_citation\&hl=en\&user=z9xw IcMAAAAJ\&citation_for_view=z9xwIcMAAAAJ:WF5omc3nYNoC

[44] Raja, M.A.Z., Khan, J.A. and Qureshi, I.M. (2010) Evolutionary Computational Intelligence in Solving the Fractional Differential Equations. Proceedings of the Asian Conference on Intelligent Information and Database Systems, Hue City, 24-26 March 2010, 231-240.

https://link.springer.com/chapter/10.1007/978-3-642-12145-6_24 https://doi.org/10.1007/978-3-642-12145-6_24

[45] Bojdi, Z.K., Ahmadi-Asland, S. and Aminataei, A. (2013) Operational Matrices with Respect to Hermite Polynomials and Their Applications in Solving Linear Differential Equations with Variable Coefficients. Journal of Linear and Topological Algebra, 2, 91-103. https://doaj.org/toc/2345-5934 
Submit or recommend next manuscript to SCIRP and we will provide best service for you:

Accepting pre-submission inquiries through Email, Facebook, LinkedIn, Twitter, etc. A wide selection of journals (inclusive of 9 subjects, more than 200 journals)

Providing 24-hour high-quality service

User-friendly online submission system

Fair and swift peer-review system

Efficient typesetting and proofreading procedure

Display of the result of downloads and visits, as well as the number of cited articles Maximum dissemination of your research work

Submit your manuscript at: http://papersubmission.scirp.org/

Or contactijmnta@scirp.org 